\title{
Modelling of lateral forces generated by pedestrians walking across footbridges
}

DOI:

10.1016/j.apm.2020.08.081

\section{Document Version}

Accepted author manuscript

Link to publication record in Manchester Research Explorer

\section{Citation for published version (APA):}

Han, H., Ding , Z., Ji, T., \& Zhang, J. (2021). Modelling of lateral forces generated by pedestrians walking across footbridges. Applied Mathematical Modelling, 89, 1775-1791. https://doi.org/10.1016/j.apm.2020.08.081

\section{Published in:}

Applied Mathematical Modelling

\section{Citing this paper}

Please note that where the full-text provided on Manchester Research Explorer is the Author Accepted Manuscript or Proof version this may differ from the final Published version. If citing, it is advised that you check and use the publisher's definitive version.

\section{General rights}

Copyright and moral rights for the publications made accessible in the Research Explorer are retained by the authors and/or other copyright owners and it is a condition of accessing publications that users recognise and abide by the legal requirements associated with these rights.

\section{Takedown policy}

If you believe that this document breaches copyright please refer to the University of Manchester's Takedown Procedures [http://man.ac.uk/04Y6Bo] or contact uml.scholarlycommunications@manchester.ac.uk providing relevant details, so we can investigate your claim.

\section{OPEN ACCESS}


Modelling of lateral forces generated by pedestrians walking across footbridges Huixuan Han ${ }^{\mathrm{a}}$, Ding Zhou ${ }^{\mathrm{b}, *}$, Tianjian Jic ${ }^{\mathrm{c}}$, Jiandong Zhang ${ }^{\mathrm{b}}$

${ }^{a}$ College of Civil Engineering and Architecture, Jiangsu University of Science and Technology, Zhenjiang 212005, China

${ }^{\mathrm{b}}$ College of Civil Engineering, Nanjing Tech University, Nanjing 211816, China

${ }^{\mathrm{c} S}$ Shool of Mechanical, Aerospace and Civil Engineering, University of Manchester, Manchester M13

$9 P L, U K$

\begin{abstract}
The mechanism which results in the synchronization of people walking across footbridges with the bridges fundamental horizontal frequency is studied. The lateral vibration of a bridge subjected to walking pedestrians is modelled by considering the bridge to be a slender beam. The effect of bridge motion on the footfall forces of walking pedestrians is not considered. The contribution of pedestrians varies with their position on the bridge. It is assumed that the walking gait frequency of a pedestrian follows a Gaussian distribution and pedestrians can unconsciously adjust the phase of their walking cycle closer to that of the bridge, based on the theory of coupled oscillators. A time-dependent nonlinear dynamic equation is derived using the modal expansion approach. The model is then applied to the north span of the Millennium Bridge in London, to produce its excessive lateral vibrations. Agreement between the results and the existing observations supports the rationality and reliability of the method. Several parameters, in particular the walking gait frequency of pedestrians, crowd sensitivity to bridge motion, bridge frequency, bridge damping and different load conditions are investigated. The numerical simulation shows that these parameters have different degrees of impact on the critical number of pedestrians triggering excessive vibrations.

Keywords: Footbridges; Lateral vibration; Lateral forces; Walking pedestrians; Synchronization

\footnotetext{
* Corresponding author.

E-mail address: dingzhou57@yahoo.com (D. Zhou)
} 


\section{Nomenclature}

\begin{tabular}{|c|c|}
\hline$A(t)$ & amplitude of lateral motion of the bridge \\
\hline$B_{j}$ & sensitivity of pedestrian to lateral motion of the bridge \\
\hline$C_{n}$ & damping coefficient of the $n$th mode of the bridge \\
\hline$c(x)$ & damping per unit length of the bridge \\
\hline$E I(x)$ & lateral flexural rigidity per unit length of the bridge \\
\hline$f_{f j}, f_{o j}$ & $\begin{array}{l}\text { lateral gait frequencies of the } j \text { th pedestrian walking forward and in the } \\
\text { opposite direction respectively }\end{array}$ \\
\hline$G$ & magnitude of lateral force generated by a pedestrian \\
\hline$H\left(t-t_{0}\right)$ & Heaviside function \\
\hline$K_{n}$ & $n$th modal stiffness of the bridge \\
\hline$L$ & length of the bridge \\
\hline$l$ & step length of the pedestrian \\
\hline$M_{n}$ & $n$th modal mass of the bridge \\
\hline$m(x)$ & mass per unit length of the bridge \\
\hline$N_{c}$ & critical number of pedestrians \\
\hline$N_{f}, N_{o}$ & $\begin{array}{l}\text { numbers of pedestrians walking forward and in the opposite direction } \\
\text { respectively }\end{array}$ \\
\hline$P_{n}(\mathrm{t})$ & $n$th lateral modal force \\
\hline$p(x, t)$ & lateral force per unit length from pedestrians \\
\hline$q_{n}(t), \dot{q}_{n}(t), \ddot{q}_{n}(t)$ & $n$th modal displacement, velocity and acceleration of the bridge \\
\hline$R$ & ramp factor \\
\hline$t$ & time \\
\hline$t_{1 j}, t_{2 j}$ & $\begin{array}{l}\text { arrival time for the } j \text { th pedestrian walking forward and in the opposite } \\
\text { direction respectively }\end{array}$ \\
\hline$t_{f j}, t_{o j}$ & $\begin{array}{l}\text { passage time for the } j \text { th pedestrian walking forward and in the opposite } \\
\text { direction respectively }\end{array}$ \\
\hline$u(x, t)$ & lateral displacement of the bridge \\
\hline$v_{f j}, v_{o j}$ & $\begin{array}{l}\text { walking speeds of the } j \text { th pedestrian walking forward and in the opposite } \\
\text { direction respectively }\end{array}$ \\
\hline$\alpha$ & phase lag between the pedestrian force and the bridge displacement. \\
\hline$\delta(x-s)$ & Dirac delta function \\
\hline$\zeta$ & damping ratio for the fundamental mode of the bridge \\
\hline$\Theta_{f j}(t), \Theta_{o j}(t)$ & $\begin{array}{l}\text { phases of the } j \text { th pedestrian walking forward and in the opposite direction } \\
\text { respectively }\end{array}$ \\
\hline$\mu, \mu_{N c}$ & mean values of gait frequencies and critical number of pedestrians respectively \\
\hline$\sigma, \sigma_{N c}$ & $\begin{array}{l}\text { standard deviations of gait frequencies and critical number of pedestrians } \\
\text { respectively }\end{array}$ \\
\hline$\Psi(t)$ & phase of the bridge \\
\hline$\phi_{n}(x)$ & $n$th vibration mode of the bridge \\
\hline$\omega_{0}$ & fundamental natural frequency of the bridge \\
\hline$\omega_{f j}, \omega_{o j}$ & $\begin{array}{l}\text { lateral angular gait frequency of the } j \text { th pedestrian walking forward and in the } \\
\text { opposite direction respectively }\end{array}$ \\
\hline
\end{tabular}




\section{Introduction}

Due to the use of new materials and modern construction technology, some footbridges are now lighter, more slender and longer than older designs. Some bridges are prone to excessive vibration when dynamically excited by pedestrians, and this may result in severe economic losses or even casualties [1]. There are several comprehensively documented cases of bridges susceptible to excessive lateral vibrations [2-6], and the measured responses of these bridges caused by pedestrians walking are generally characterized by a rapid onset of vibration following a small increase in crowd numbers.

The notorious behavior of the London Millennium Footbridge (LMF) on its opening day, generated a large amount of research interest in human-structure dynamic interaction [6-9]. Biomechanicists have been mainly concerned with adjustments in walking gait parameters, while civil engineers have mostly focused on quantification of changes of human-induced forces, or the additional mass and damping from occupants. For pedestrians walking on a laterally flexible footbridge, modelling of human-induced loading and its effect on footbridges has drawn considerable attention $[3,10-12]$. In recent years, many lateral human force models in biomechanics (such as inverted pendulum models) [13-17] have been proposed to explore the mechanics of human balance during walking and energy exchange between a crowd and a moving surface. Laterally oscillating treadmills [18-21] and footbridges in controlled laboratory conditions [22] have been used to measure pedestrian forces. In addition, wearable sensors [23, 24] have been used as a proxy 
due to the lack of direct measurements on full-scale footbridges. However, the results rely mainly on the correct alignment of the data collected from these sensors.

To date, the most popular assumed cause of excessive lateral vibration induced by walking traffic has been the synchronized action of walking pedestrians. Two categories of synchronization mechanisms have been suggested, namely, human-to-structure synchronization (synchronous lateral excitation (SLE) or pedestrian lock-in) and human-to-human synchronization. The former mechanism is explained as pedestrians tend to synchronize with the movement of the structure, adjusting the frequency and phase of their footsteps in a manner which increases the bridge motion. The latter mechanism is that synchronization between individuals may be promoted due to the effects of many factors such as space and psychology. Many models based on the synchronization paradigm have been proposed [2,6, 25-29]. Pedestrian walking was modelled as a harmonic force by Fujino et al. [2] to explain the lateral vibration of the Toda Park Bridge with a parameter empirically tuned according to their experimental results. Pedestrians were treated as negative dampers by Dallard et al. [6] and Nakamura [25], which essentially amplified bridge response. However, their loading models were empirical due to the lack of generality for parameter determination. Newland [26] developed a model based on the synchronization phenomenon widely studied in wind engineering. In his model, the lateral force was expressed as the sum of two terms, namely, the force exerted by pedestrians on a fixed ground and the component caused by the moving surface. His model included the assumption that $40 \%$ of the pedestrians were synchronized with 
the bridge's lateral frequency, independent of the amplitude of bridge motion, but this cannot account for the inter-subject variability of the pedestrians. The model proposed by Roberts [27] assumed that the pedestrians would synchronize so as to destabilize the bridge, but did not account for the onset of synchronization. A quite different model was proposed by Strogatz et al. [28,29] adopting ideas from some well-known examples of collective synchronization in the physics of life. They believed that existing theories mostly focus on the sway of the bridge without addressing the crowd-synchronization dynamics sufficiently, which are inseparable with the sway. Based on the controlled tests [30] on the LMF, Strogatz model reasonably explained the simultaneous growth of bridge motion and pedestrians synchronization. However, the lateral forces in their model were assumed as concentrated forces on the midspan of the footbridge, neglecting the influence of the space and time combinations from pedestrians. More recently, a linear mathematical model of synchronization of groups of people performing cyclic motion, such as walking, taking into account the interaction between the individuals, has been proposed by Gazzola and Racic [31], but this is limited to small movements of the structure. Toso et al. [32] have used a fully synchronized force model, in longitudinal and lateral direction of pedestrian movement, combined with a biodynamic model in vertical direction to describe walking forces. Their model allows the assessment of the structural behavior in all directions, although the excessive vibration of the footbridge cannot be simulated.

Most of the load models reviewed above do not take into account the time-varying forces produced by pedestrians. Generally, only unidirectional traffic has been 
considered in these models, which is not realistic. Therefore, inspired by Strogatz's approach, a more refined model is proposed herein to describe the lateral vibration of footbridges subjected to bi-directional walking pedestrians. Because the total mass of pedestrians on a bridge is much smaller than the mass of the bridge, walking pedestrians will not significantly change the modal properties of the bridge. Therefore, in the present study, the effects of walking pedestrians are only considered as lateral footstep forces acting on the bridge. The inter-subject variability is considered by introducing probabilistic parameters that mainly affect the human force, such as walking frequency. The remainder of this paper is organized as follows. Section 2 outlines the theoretical model for the interacting pedestrian-bridge system. The validation of the present model is illustrated with reference to the real case of the LMF in Section 3. Parametric investigations are presented in detail in Section 4 and the conclusions are given in Section 5.

\section{Analytical model}

A footbridge excited by bi-directional walking pedestrians is shown in Fig. 1. The bridge is considered as a slender beam with length $L$, mass per unit length $m(x)$, damping per unit length $c(x)$ and flexural rigidity of cross-section $E I(x)$, which is mainly subjected to bending deformation. Ignoring the effect of shear deformation will not produce a great error. Pedestrians enter from the two ends of the bridge on their left side. Therefore, for this exercise, there will be no collision between pedestrians walking in opposite directions. The lateral displacement of the bridge in the $y$ direction is denoted by $u(x, t)$. The lateral force per unit length along the bridge, 
exerted by bi-directional walking pedestrians, is denoted by $p(x, t)$.

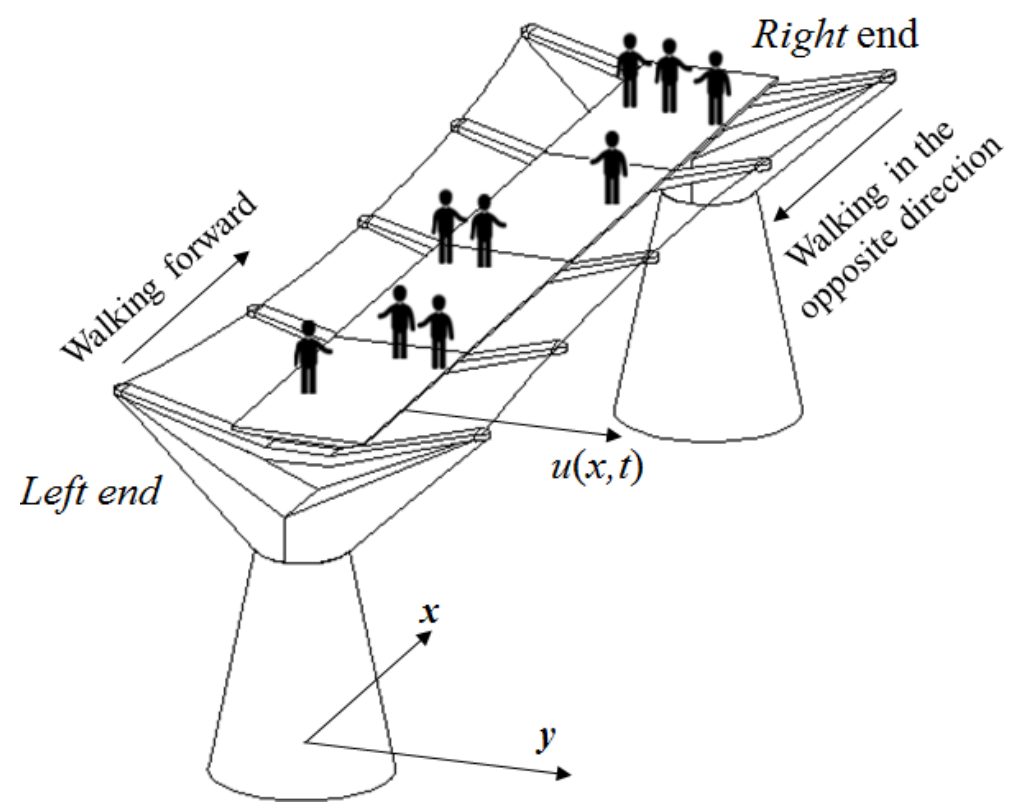

Fig. 1. Pedestrian-bridge interaction system subjected to bi-directional walking.

The governing differential equation of the bridge lateral motion can be written as:

$$
\frac{\partial^{2}}{\partial x^{2}}\left[E I(x) \frac{\partial^{2} u(x, t)}{\partial x^{2}}\right]+m(x) \frac{\partial^{2} u(x, t)}{\partial t^{2}}+c(x) \frac{\partial u(x, t)}{\partial t}=p(x, t)
$$

Due to the intra-subject variability, the measured individual lateral walking forces from experiments $[18,23,33]$ were invariably near-periodic with a narrow-band nature. It is assumed that continuous walking can be obtained from a succession of identical footsteps, as shown in Fig. 2. It is seen that the time period $T_{\text {lateral }}$ is equal to the time of a full left-right walking cycle. The first term in the Fourier series expansion of a generic periodic function is used to represent the lateral force for each pedestrian. This is because the first term is the main term which reflects the key effect of the force. 


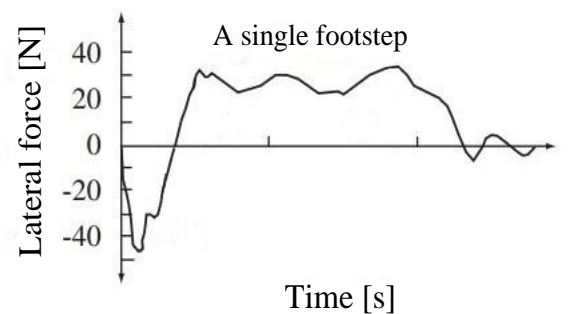

(a)

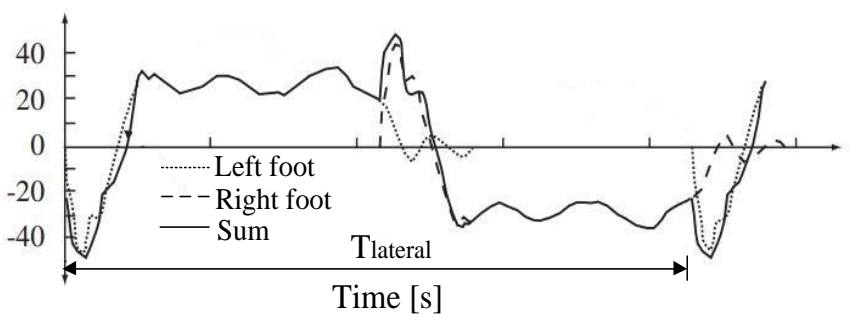

(b)

Fig. 2. Typical shape of walking force in lateral direction: (a) a single footstep and (b) a series of continuous footsteps (figure reproduced from [18]).

Strictly speaking, the human-structure system is a time-varying system, and the lateral force exerted by each pedestrian on the bridge varies with his/her walking position. Therefore, the Dirac delta function and the Heaviside function are both introduced to describe the force generated by each pedestrian:

$$
\begin{aligned}
& \delta(x-s)= \begin{cases}1 & x=s \\
0 & x \neq s\end{cases} \\
& H\left(t-t_{0}\right)= \begin{cases}0 & t<t_{0} \\
1 & t \geq t_{0}\end{cases}
\end{aligned}
$$

where $s$ and $t_{0}$ are the pedestrian walking position and arrival time, respectively.

It is assumed that each pedestrian walks straight along the bridge at a constant speed which varies for different pedestrians. It should be noted that the contribution of each pedestrian is viewed as an imposed load. The effect of bridge motion on the pedestrian footstep force is not considered. The numbers of pedestrians with different moving directions are given as $N_{f}$ and $N_{o}$. Subscripts " $f$ " and " $o$ " indicating moving from left end of the bridge to the right (denoted as "walking forward") and in the opposite direction, are used in all the following formulae. Then, the spatial distribution of the pedestrian force, as a sum of the moving concentrated forces exerted by bi-directional walking pedestrians, can be expressed as: 
$p(x, t)=p_{f}(x, t)+p_{o}(x, t)$

$=G \sum_{j=1}^{N_{f}} \delta\left(x-v_{f j}\left(t-t_{1 j}\right)\right) \varepsilon_{f j}(t) \sin \Theta_{f j}(t)+G \sum_{j=1}^{N_{o}} \delta\left[x-\left(L-v_{o j}\left(t-t_{2 j}\right)\right)\right] \varepsilon_{o j}(t) \sin \Theta_{o j}(t)$

in which,

$t_{f j}=L / v_{f j}, t_{o j}=L / v_{o j}$

$\varepsilon_{f j}(t)=H\left(t-t_{1 j}\right)-H\left(t-t_{1 j}-t_{f j}\right)$

$\varepsilon_{o j}(t)=H\left(t-t_{2 j}\right)-H\left(t-t_{2 j}-t_{o j}\right)$

where $G$ is the amplitude of the sideways force generated by a single pedestrian and is assumed to be the same for all pedestrians. $t_{f j}$ (or $t_{o j}$ ) is the passage time for the $j$ th pedestrian walking forward (or in the opposite direction). $t_{1 j}$ and $t_{2 j}$ are the arrival time for the $j$ th pedestrian with different moving directions, respectively. $\Theta_{f j}(t)\left(\right.$ or $\left.\Theta_{o j}(t)\right)$ is the phase in the walking cycle for pedestrian $j$ walking forward (or in the opposite direction). It is assumed that $\Theta_{f j}=\Theta_{o j}=0$ when the pedestrian's left foot touches the ground, and $\Theta_{f j}=\Theta_{o j}=\pi$ when the right foot touches the ground, as shown in Fig. 3. It should be noted that the phase $\Theta_{f j}(t)$ and $\Theta_{o j}(t)$ increase by $2 \pi$ during a full left-right walking cycle.

It is assumed that a pedestrian $j$ walks forward (or in the opposite direction) with an angular gait frequency $\omega_{f j}$ (or $\omega_{o j}$ ) in the absence of bridge motion. The corresponding gait frequency is $f_{f j}$ (or $f_{o j}$ ). There is a physical relationship between the walking speed $v_{f j}$ (or $v_{o j}$ ) and the step length $l_{f j}\left(\right.$ or $\left.l_{o j}\right)$ in terms of the gait frequency as $v_{f j}=2 l_{f j} f_{f j}, \quad v_{o j}=2 l_{o j} f_{o j}$

The geometric displacement of the bridge in the $y$ direction can be obtained using a modal superposition approach: 
$u(x, t)=\sum_{n=1}^{\infty} \phi_{n}(x) q_{n}(t)$

where $\phi_{n}(x)$ is the $n$th vibration mode of the bridge and $q_{n}(t)$ is the generalized coordinate of the mode [34].

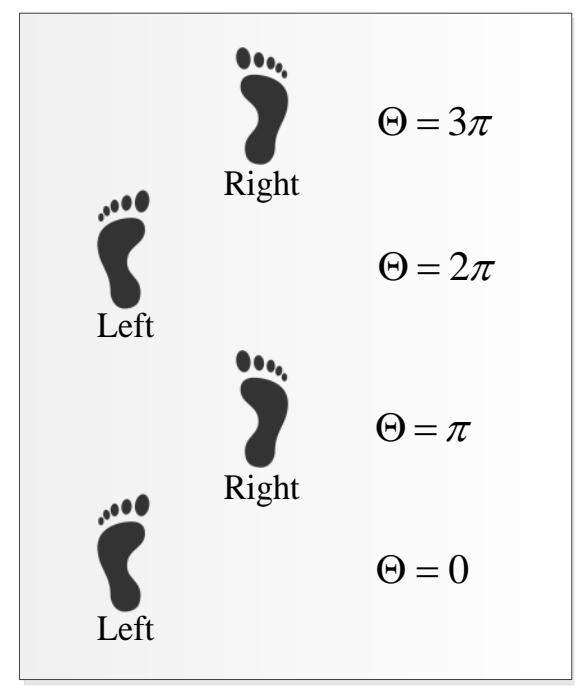

Fig. 3. Diagrammatic representation of $\Theta$.

Based on the orthogonality of modes, the governing differential equation of the bridge movement can be written by substituting Eqs. (5)-(6) into Eq. (1), multiplying by $\phi_{m}(t)$, and integrating over the length of the bridge $L$, as follows:

$M_{n} \ddot{q}_{n}(t)+C_{n} \dot{q}_{n}(t)+K_{n} q_{n}(t)=P_{n}(t), \quad(n=1,2,3, \ldots)$

where the overdots in Eq. (7) refer to differentiation with respect to time $t$; the $n$th modal characteristics of the footbridge $M_{n}, C_{n}$ and $K_{n}$ are

$$
M_{n}=\int_{0}^{L} m(x) \phi_{n}^{2}(x) d x, \quad C_{n}=\int_{0}^{L} c(x) \phi_{n}^{2}(x) d x, \quad K_{n}=\int_{0}^{L} \phi_{n}(x) \frac{\partial^{2}}{\partial x^{2}}\left[E I(x) \frac{\partial^{2} \phi_{n}(x)}{\partial x^{2}}\right] d x
$$

and the lateral modal pedestrian force $P_{n}$ is

$$
P_{n}(t)=\int_{0}^{L} \phi_{n}(x) p(x, t) d x=G \sum_{j=1}^{N_{f}} \phi_{n}\left(x_{f j}\right) \varepsilon_{f j}(t) \sin \Theta_{f j}(t)+G \sum_{j=1}^{N_{o}} \phi_{n}\left(x_{o j}\right) \varepsilon_{o j}(t) \sin \Theta_{o j}(t)
$$

in which 
$x_{f j}=2 l_{f j} f_{f j}\left(t-t_{1 j}\right), \quad x_{o j}=L-2 l_{o j} f_{o j}\left(t-t_{2 j}\right)$

When two pedestrians walking in opposite directions meet, their contribution to lateral modal pedestrian forces may cancel if their relative phases differ by 180 degrees. If they share the same phase value, their contributions will add up.

According to kinetic theory, the structural fundamental vibration mode generally contributes significantly to the response. Here the higher modes of the bridge are ignored because, in general, their frequencies are far higher than the human pacing rate which leads to a negligible effect. Then, the governing differential equation for the bridge can be simplified to be

$M_{1} \ddot{q}_{1}(t)+C_{1} \dot{q}_{1}(t)+K_{1} q_{1}(t)=P_{1}(t)$

where

$P_{1}(t)=G \sum_{j=1}^{N_{f}} \phi_{1}\left(x_{f j}\right) \varepsilon_{f j}(t) \sin \Theta_{f j}(t)+G \sum_{j=1}^{N_{o}} \phi_{1}\left(x_{o j}\right) \varepsilon_{o j}(t) \sin \Theta_{o j}(t)$

Some experimental results $[18,21]$ suggest that the tendency for pedestrians to synchronize with lateral structural motion is too weak for the development of excessive lateral vibrations, while some investigators [35] observed strong synchronization phenomenon from experiments. In the present study, the latter view is accepted. Phase synchronization, originating from the theory of coupled oscillators [28-29], is adopted herein to describe the pedestrians-bridge interaction as follows:

$\dot{\Theta}_{j}(t)=\omega_{j}+B_{j} A(t) \sin \left(\Psi(t)-\Theta_{j}(t)+\alpha\right)$

where $\omega_{j}$ and $\Theta_{j}(t)$ are the angular walking gait frequency on the bridge, with a random distribution and the phase for each of the $\left(N_{f}+N_{o}\right)$ pedestrians. $A(t)$ and $\Psi(t)$ are the amplitude and phase of the bridge. $\alpha$ is a constant phase lag which denotes the 
initial phases between the human-induced force and the displacement of the bridge. $B_{j}$, with units of angle/distance per unit time, quantifies the pedestrian sensitivity to the amplitude of the bridge vibration $A(t)$. It is assumed that each pedestrian's gait changes as a result of the bridge's movement. The term $f=B_{j} A(t) \sin \left(\Psi(t)-\Theta_{j}(t)+\alpha\right)$ is chosen to be a function of the bridge motion amplitude, the bridge phase and the pedestrian phase. $f$ has the effect of shifting pedestrians to a phase closer to that of the bridge, which can describe the natural tendency of the pedestrian-bridge system to synchronize. When $\Theta_{j}$ lags $\Psi+\alpha, f$ is positive and increases the pedestrian frequency $\dot{\Theta}_{j}$. Conversely, when $\Theta_{j}$ leads $\Psi+\alpha, f$ is negative and decreases $\dot{\Theta}_{j}$ in order to lock on to the bridge motion. This relationship is assumed to be in the form of a sinusoidal function as the simplest periodic function that satisfies the synchronization requirements. From another point of view, this can be interpreted as the first term in a Fourier expansion of the periodic function $f$. It is considered in this force model that the gait frequencies of pedestrians, which are initially random, become locked into the bridge frequency (namely phase synchronization) if the vibration amplitude is sufficiently strong.

In Eqs. (9) and (11), unknowns $q_{1}(t)$ and $\Theta_{j}(t)$ are obtained through integration. It is complicated to define the variables $A(t)$ and $\Psi(t)$ in the transient state, therefore, at least as far as steady state is concerned, they are given by:

$$
\sin \Psi=q_{1} / A, \quad \cos \Psi=\dot{q}_{1} /\left(\omega_{0} A\right), \quad A=\sqrt{q_{1}^{2}+\frac{\dot{q}_{1}^{2}}{\omega_{0}^{2}}}
$$

where $\omega_{0}=\sqrt{K_{1} / M_{1}}$ is the angular lateral fundamental frequency of the bridge.

A dummy variable $y_{1}$ is introduced. Eq. (9) is converted into a system of first order 
equations to integrate as follows:

$\dot{q}_{1}(t)=y_{1}$

$\dot{y}_{1}=\frac{P_{1}(t)}{M_{1}}-\frac{C_{1}}{M_{1}} y_{1}-\frac{K_{1}}{M_{1}} q_{1}(t)$

The governing differential equations of walking pedestrian-footbridge interaction can be rewritten by expanding the sine function in Eq. (11) and substituting Eq. (12) into Eq. (11) to replace $A(t)$ and $\Psi(t)$ as

$\dot{\Theta}_{j}(t)=\omega_{j}+B_{j}\left[q_{1}(t) \cos \left(\Theta_{j}(t)-\alpha\right)-\frac{y_{1}}{\omega_{0}} \sin \left(\Theta_{j}(t)-\alpha\right)\right]$

\section{Validation of the model}

The validation of the model is illustrated through numerical response simulations, which employs the fundamental mode of the LMF. This example has been selected because the bridge has been investigated intensively in the past decade. In particular the experimental results of the north span of the bridge [6] are considered herein. The bridge is considered to be a uniform simple beam. For this representation, the fundamental modal function, either for a Euler beam or a Timoshenko beam, can be expressed as

$\phi_{1}(x)=\sin \frac{\pi x}{L}$

The basic data from the LMF used for this analysis are: span $L=81.0 \mathrm{~m}$, mass per meter of its length $m=2000 \mathrm{~kg} / \mathrm{m}$, the fundamental lateral natural frequency $\omega_{0}=6.47$ $\mathrm{rad} / \mathrm{s}$ (corresponding to $1.03 \mathrm{~Hz}$ ) and damping ratio $\zeta=0.75 \%$ [6,28]. The modal mass $M_{1}$, modal stiffness $K_{1}$ and modal damping $C_{1}$ can be estimated:

$$
M_{1}=\int_{0}^{L} m \sin ^{2}(\pi x / L) L d x=m L / 2=2000 \times 81 / 2=81000 \mathrm{~kg}
$$




$$
\begin{aligned}
& K_{1}=M_{1} \omega_{0}^{2}=81000 \times 6.47^{2}=3390733 \mathrm{~kg} / \mathrm{s}^{2} \\
& C_{1}=2 \zeta M_{1} \omega_{0}=2 \times 0.75 \times 81000 \times 6.47=7681 \mathrm{~kg} / \mathrm{s}
\end{aligned}
$$

The value of $G$ used in the simulations is $30 \mathrm{~N}$, which corresponds to the mean amplitude of the measured lateral force exerted by one pedestrian on a stationary surface $[3,10]$. The pedestrian sensitivity coefficient $B_{j}=B=16 \mathrm{~m}^{-1} \mathrm{~s}^{-1}$ has been estimated from experimental results [28-29] due to the lack of further detailed data. Some experimental results have shown that gait frequencies for pedestrians during unrestrained walking are between $0.7 \mathrm{~Hz}$ and $1.2 \mathrm{~Hz}$ [11,18,36-37]. In order to verify this model, a Gaussian distribution with mean $\mu=1.03 \mathrm{~Hz}$ and standard deviation $\sigma=0.1 \mathrm{~Hz}$, consistent with reference [28], is adopted for gait frequencies $\omega_{j} . \alpha=\pi / 2$ is a worst-case scenario for the input lateral force which is in phase with the modal velocity. In this special case, the bridge is maximally destabilized, which leads to a conservative prediction of the critical number of pedestrians. The initial phase $\Theta_{j}$ for each pedestrian is assumed uniformly distributed in the interval $[0,2 \pi][28]$.

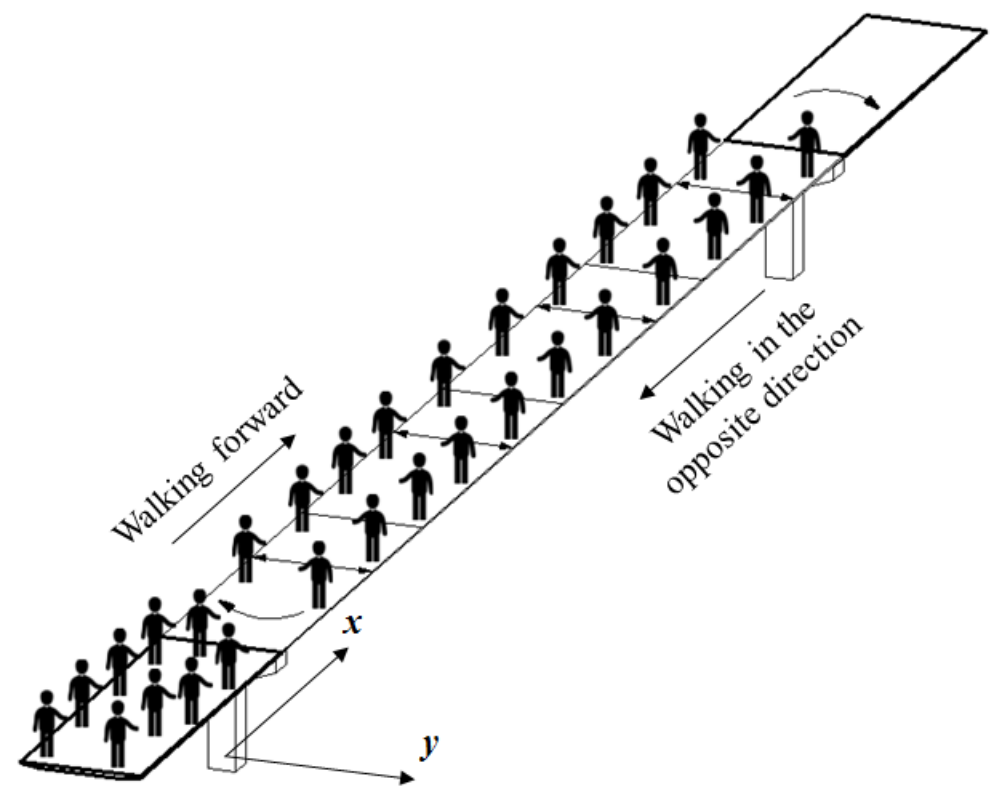

Fig. 4. A diagram showing pedestrians walking back and forth on the bridge. 


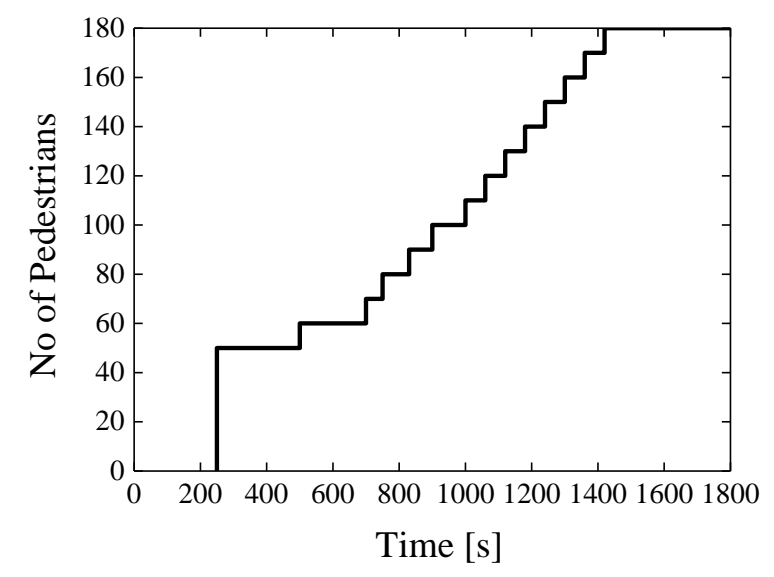

Fig. 5. The loading path of numbers of pedestrians on the bridge.

Based on video footage recorded during Arup's test on site $[6,30]$, pedestrians are considered to walk back and forth between two ends of the bridge, as shown in Fig. 4. A time history of pedestrian participation given by Marcheggiani and Lenci [38] is shown in Fig. 5. The number of pedestrians gradually increases from 50 to 180, by 10 people at each time step. The values of the initial displacement and velocity of the bridge are assumed to be zero.

In general, it is realistic to consider the variability of the step lengths of individuals by using a random distribution. However, for the sake of simplicity, a common constant step length $l$ for all pedestrians is used in this study to demonstrate some basic features of the present model. Three values $l=0.35 \mathrm{~m}, 0.50 \mathrm{~m}$ and $0.70 \mathrm{~m}$ representing $50 \%, 70 \%$ and $100 \%$ of the statistical value of step length determined from normal walking pedestrians given by Pachhi and Ji [36], are considered herein. The numerical simulation of the present model of the LMF is carried out using Matlab with the solver function ODE45. 


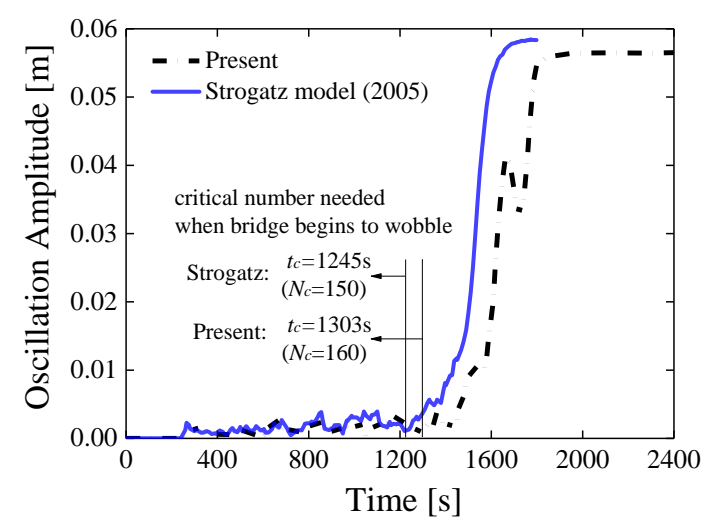

(a)

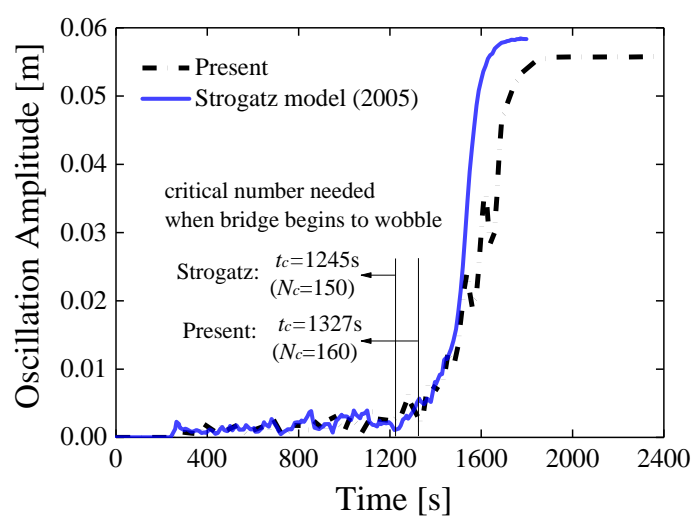

(b)

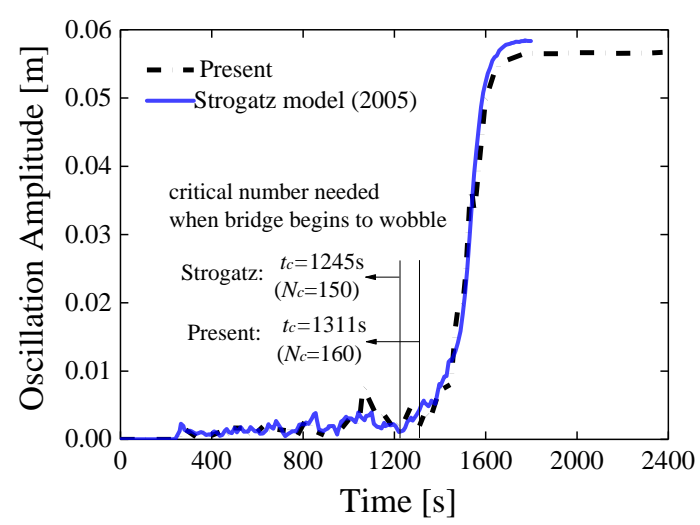

(c)

Fig. 6. Comparisons of the displacement amplitude time histories at the mid-span between the present model at three different pedestrian step lengths and the Strogatz model: (a) $l=0.35 \mathrm{~m}$, (b) $l=0.50 \mathrm{~m}$, and (c) $l=0.70 \mathrm{~m}$.

Fig. 6 shows typical plots of lateral displacement amplitude time histories at the mid-span of the bridge adopting the Strogatz model [28] and the present model for the three different step lengths without changing the gait frequencies of the pedestrians. It can be seen that the time histories for the three step lengths are similar in shape. When the number of pedestrians on the bridge is small, the oscillation amplitudes of the three step lengths are close to zero, with small fluctuations resulting from the randomness in the pedestrian force coefficients. When the number increases to 160 , as shown in Fig. 6, the bridge begins to sway. As the crowd number further increases, the oscillation amplitudes remains stable. The maximum final amplitudes of the bridge at 
three step lengths obtained from Fig. 6 are $0.05641,0.05581$ and $0.05665 \mathrm{~m}$. It is observed that the steady-state amplitudes of the three step lengths are almost identical. However, as the step length (or walking speed) increases, the time to reach the steady-state amplitude decreases. There is around a 200 second difference between the time at which this is reached in Fig. 6(a) and that in Fig. 6(b). The value 160 is defined as the critical threshold of instability, after which wobbling and synchrony suddenly occur. In this analysis, the critical value $N_{c}$ is estimated based on the identification of the onset for the exponential growth of the instability $t_{c}$. Three straight segments are used to fit the displacement amplitude history curves. When the slope of the second segment increases significantly, the intersection point between the first and the second segment corresponds to the onset time $t_{c}$. It should be noted that although the onset time $t_{c}$ of excessive lateral vibration is slightly different as step length varies, the corresponding critical numbers $N_{c}$ are the same.

From Fig. 6, it can be seen that the Strogatz model shows similar qualitative behavior to the present model. For small crowds, the two models give similar predictions with fluctuating results. However, when the number of pedestrians increases, the Strogatz model shows a monotonic increase of amplitude while the present model gives a slight oscillatory increase of amplitude. The critical number from the Strogatz model is 150 , which is smaller than the present model. It is clearly seen that the steady-state amplitude of the Strogatz model is slightly larger than that of the present model for different step lengths. However, the time at which this amplitude is reached is different in the two models, with a maximum discrepancy of 
about 200 seconds, as shown in Fig. 6(a).

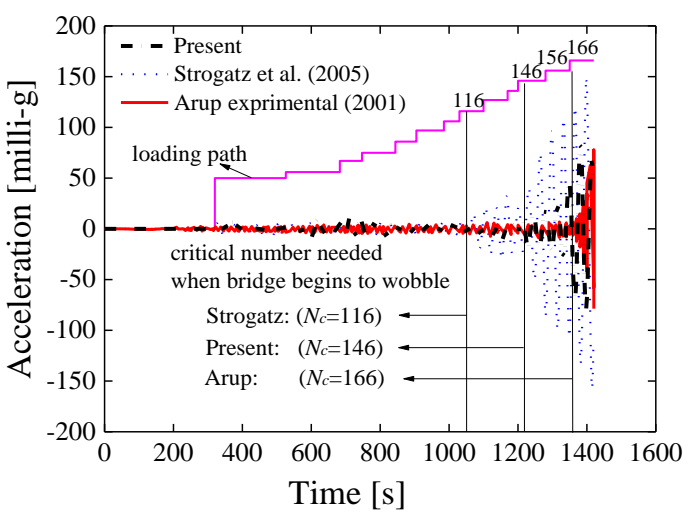

(a)

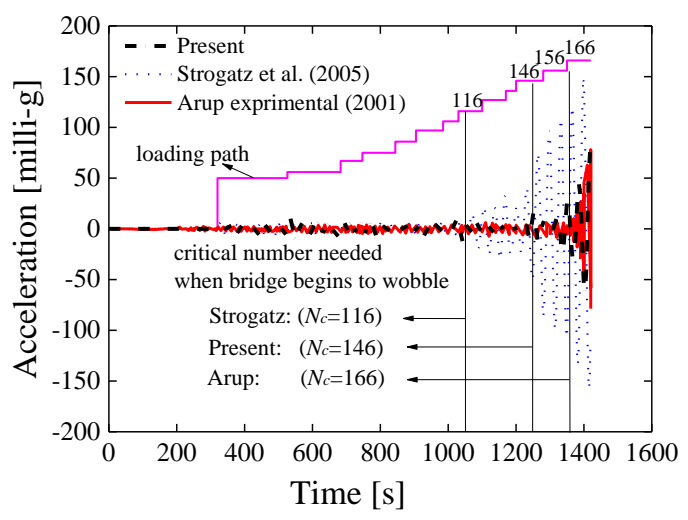

(b)

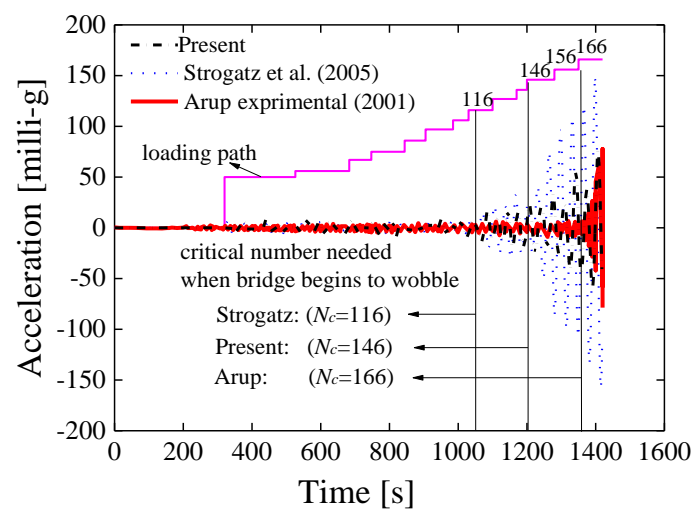

(c)

Fig. 7. Comparisons of the lateral acceleration time histories at the mid-span of the bridge showing Arup's measurements [39], Strogatz model [28] and the present model at three different step lengths: (a) $l=0.35 \mathrm{~m}$, (b) $l=0.50 \mathrm{~m}$, and (c) $l=0.70 \mathrm{~m}$.

Fig. 7 shows the mid-span lateral acceleration time histories of the bridge for Arup's measurements [39], Strogatz model [28] and the present model at three step lengths. The loading path in Fig. 7 illustrates the relationship between the number of pedestrians involved and the entry time measured during Arup's tests on the north span of the LMF [39]. It should be noted that Strogatz et al. only provided the displacement response of the north span. Therefore, the acceleration response is simulated based on their model according to the loading path given in Fig. 7.

It can be seen, from the responses using the present model, that there is generally 
no substantial difference for the three step lengths. For small crowds, the oscillation amplitudes at the three step lengths are near zero with small fluctuations. When the crowd reaches a critical number $N_{c}=146$, the bridge begins to wobble. It is observed that the critical thresholds of the three step lengths are almost identical, although the onset time $t_{c}$ for excessive lateral vibration varies slightly. The maximum accelerations of the bridge at the three step lengths are almost identical and the values detected from Fig. 7 are 83.35, 75.71 and 75.45 milli-g.

From Fig. 7, it can be seen that the lateral accelerations of the two models both show that a small increase beyond a critical number of pedestrians $\left(N_{c}=116\right.$ for Strogatz model and $N_{c}=146$ for the present model) results in diverging response amplitudes. The critical number detected from Arup's test results was $N_{c}=166$. The difference of the critical values $N_{c}$ between the two models comes mainly from the pedestrian-induced lateral force. The contribution of each pedestrian is considered as a concentrated force at the mid-span of the bridge in the Strogatz model. The effect of each pedestrian's walking position on the lateral force, which was neglected in Strogatz model, is considered in the present model. Both models include some simplifications which may result in the difference between the critical values $N_{c}$ of either model and the Arup's measurements. The maximum accelerations of the Strogatz model and the experimental measurements are 157.23 and 78.70 milli-g. Obviously, both the critical threshold and the maximum acceleration of the present model appear closer to Arup's measurements than those of the Strogatz model. 


\section{Parametric investigations}

Several parameters which may mainly affect the present model behavior are chosen to give a deeper insight into the SLE phenomenon. The performance of the present model is also demonstrated through numerical response simulations employing the fundamental mode of the north span of the LMF to serve as a suitable platform for comparison with other models and measurements in the future.

During the opening day of the LMF, the maximum density of the pedestrians was estimated as being between 1.3 and 1.5 people per square meter. Therefore, in the following simulations, the maximum crowd size is considered to be 486 (at a density of 1.5 persons $/ \mathrm{m}^{2}$ over the north span).

Two criteria for estimating the critical number of pedestrians $N_{c}$ are considered in this section. Criterion 1 is defined by the trigger of the SLE phenomenon to identify the onset time $t_{c}$ after which wobbling and synchrony suddenly emerge. Criterion 2 is defined by the tolerance level of human perception of vibrations when the lateral acceleration exceeds a threshold of $0.2 \mathrm{~m} / \mathrm{s}^{2}$, according to Setra [40].

Due to the randomness of the initial conditions assigned to pedestrians, each simulation has been repeated 100 times. Therefore, the critical number of pedestrians $N_{c}$ is randomly distributed with a mean value $\mu_{N c}$ and standard deviation $\sigma_{N c}$.

\subsection{Effect of different load events}

Nine linear loading paths, which differ in the combinations of step size and the duration of the load events, are studied here to investigate the variations of the critical threshold. In the following simulations, the crowd size is gradually increased from 0 
to 486 pedestrians and the step length $l$ is considered to be $0.5 \mathrm{~m}$. Each simulation has been repeated 100 times.

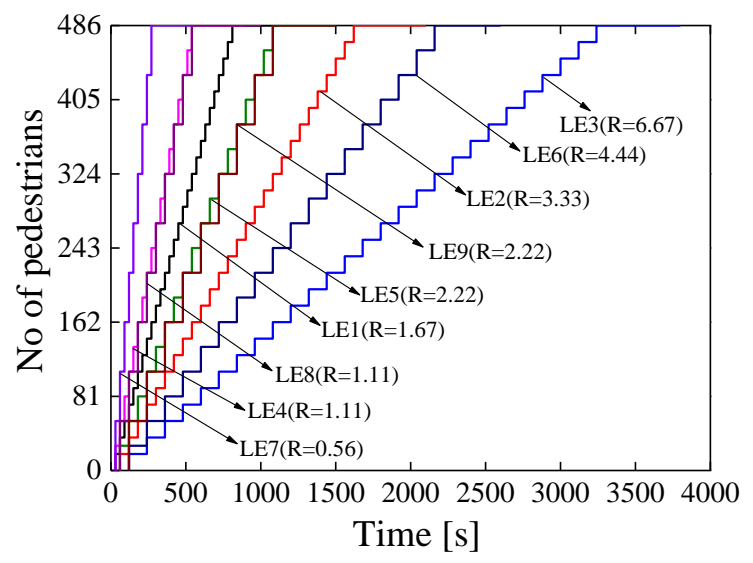

Fig. 8. Loading paths for the nine load events.

Fig. 8 shows the nine linear loading paths, with the slope of each path representing the number of pedestrians introduced on the bridge deck per unit time. A ramp factor $R$ is introduced to represent the reciprocal of the slope in Fig. 8. These load events have been divided into three groups which are listed as follows:

LE1: in steps of 18 every half minute $(R=30$ seconds $/ 18$ pedestrians $=1.67)$;

LE2: in steps of 18 every 1 minute $(R=60$ seconds $/ 18$ pedestrians $=3.33)$;

LE3: in steps of 18 every 2 minutes $(R=120$ seconds/18 pedestrians $=6.67)$;

LE4: in steps of 27 every half minute $(R=1.11)$;

LE5: in steps of 27 every 1 minute $(R=2.22)$;

LE6: in steps of 27 every 2 minutes $(R=4.44)$;

LE7: in steps of 54 every half minute $(R=0.56)$;

LE8: in steps of 54 every 1 minute $(R=1.11)$;

LE9: in steps of 54 every 2 minutes $(R=2.22)$;

It is seen from Fig. 8 that LE4 in G2 and LE8 in G3 share the same $R$ value of 1.11. 
And the $R$ values of LE5 in G2 and LE9 in G3 are identical.

Fig. 9 gives the mean values of critical numbers for different load events under two criteria. From the figure, it can be seen that an increase in the duration of the simulation within each group, leads to a decrease of $\mu_{N_{c}}$ for the two criteria. There is also a trend that an increase in the step of the simulation between the three groups has the effect of increasing $\mu_{N_{c}}$ for both criteria. The crowd critical size $\mu_{N_{c}}$ of Criterion 2 is slightly larger than the corresponding value of Criterion 1. It is noteworthy that LE4 and LE8, which share the identical $R$ value, have the same critical value $\mu_{N c}$. This also applies to LE5 and LE9.

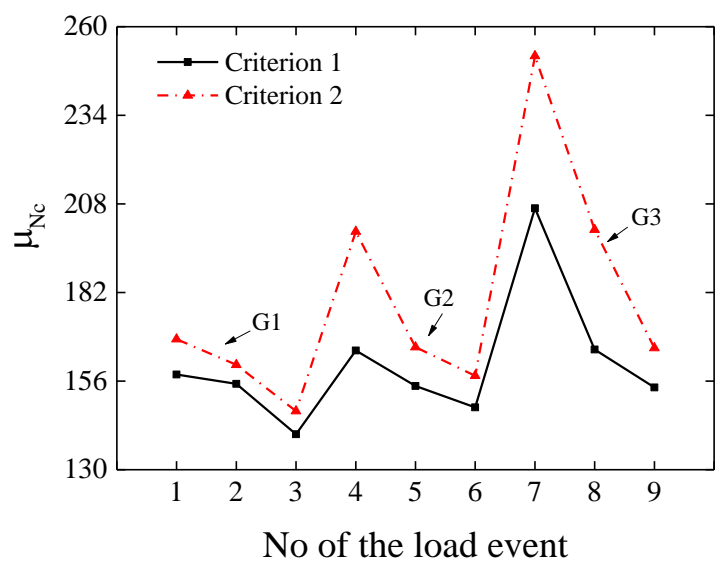

Fig. 9. The mean values of critical numbers of nine different load events for the two criteria.

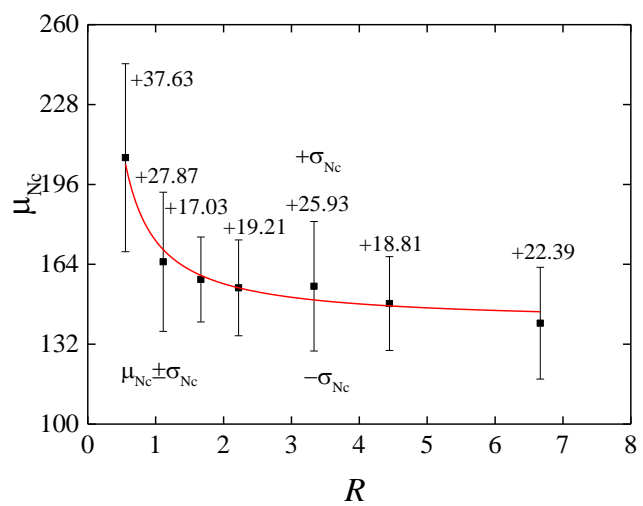

(a)

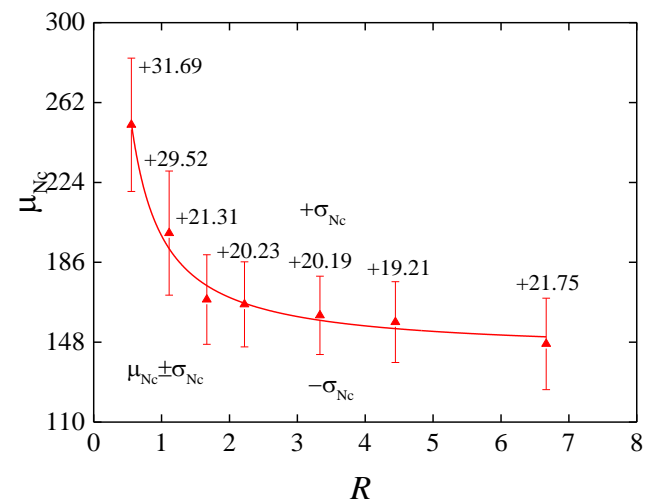

(b)

Fig. 10. Critical numbers of pedestrians as functions of the ramp factor for: (a) Criterion 1, and (b) Criterion 2. 
Fig. 10 shows the mean values $\mu_{N c}$ of the critical crowd numbers for both criteria as functions of the ramp factor $R$. The fitted exponential decay curves through the mean values $\mu_{N c}$, and the standard deviations $\sigma_{N c}$, are also shown in Fig. 10. The corresponding best-fit functions for the two criteria are given as follows:

$$
\begin{aligned}
& \mu_{N c 1}=140.4 \exp \left(\frac{0.21584}{R+0.02007}\right) \\
& \mu_{N c 2}=142.96321 \exp \left(\frac{0.34814}{R+0.05844}\right)
\end{aligned}
$$

As expected, it is observed that $\mu_{N c}$ for either wobbling, or in excess of the acceleration threshold $0.2 \mathrm{~m} / \mathrm{s}^{2}$, generally decreases with an increase of the ramp factor $R$. And the curve trends for the mean value $\mu_{N_{c}}$ for both criteria become steady when the ramp factor $R$ increases to certain values. In fact, for low $R$ values, the pedestrian-bridge system does not have enough time to develop synchronization, leading to an increase of the crowd critical size. For high $R$ values, the bridge has enough time to become unstable and pedestrians are soon driven so that their footsteps lock into the bridge motion. Consequently, the critical crowd size for high $R$ values is lower than that for low $R$ values.

The critical number decreases as the duration of the simulation increases, which is consistent with the predictions given by Ingólfsson and Georgakis [41]. The critical number becomes steady when the ramp factor $R$ increases to around 6 , which corresponds to a similar slope of the Arup's ramp after the initial transient [39]. This means that the Arup's critical threshold can be a conservative estimation and a lower bound for other loading events. 


\subsection{Effect of crowd initial phase distribution}

The crowd size is gradually increased from 0 to 486 pedestrians in steps of 18 every 1 minute. The step length $l$ is considered to be $0.5 \mathrm{~m}$. The initial phases of pedestrians are considered uniformly distributed in three different intervals, namely $[0,2 \pi],[0, \pi]$ and $[0,1]$, respectively.

Fig. 11 shows typical plots of the maximum lateral displacement and acceleration time-histories at the mid-span for three different intervals initial phase distributions for the pedestrians. It can be observed that no substantial difference exists between these three intervals.

Table 1 gives the mean values $\mu_{N c}$ and standard deviations $\sigma_{N c}$ of the critical thresholds for the three distributed intervals of initial phases for the two different criteria. It is seen both criteria have almost the same average critical values with similar standard deviations. When the distributed interval is narrowed, which means the initial behavior is less random among the pedestrians, the standard deviation $\sigma_{N c}$ decreases. Actually, low standard deviation $\sigma_{N c}$ indicates that pedestrians synchronize quicker. The crowd critical size $\mu_{N c}$ for Criterion 2 is slightly larger than the corresponding value for Criterion 1 .

From Fig. 11 and Table 1, it can be concluded that the level of phase synchrony among pedestrians has little influence on the dynamic responses of the bridge, due to pedestrians' different normal gait frequencies. The onset of the phenomenon is not affected by initial phase distributions, which shares a similar conclusion with [38]. 


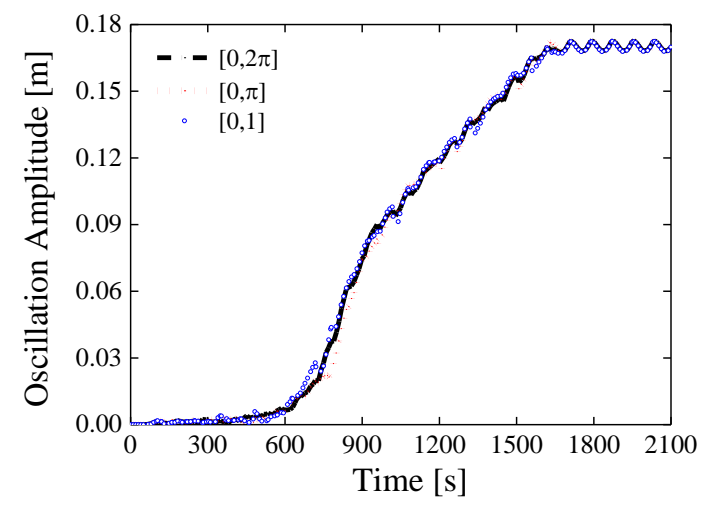

(a)

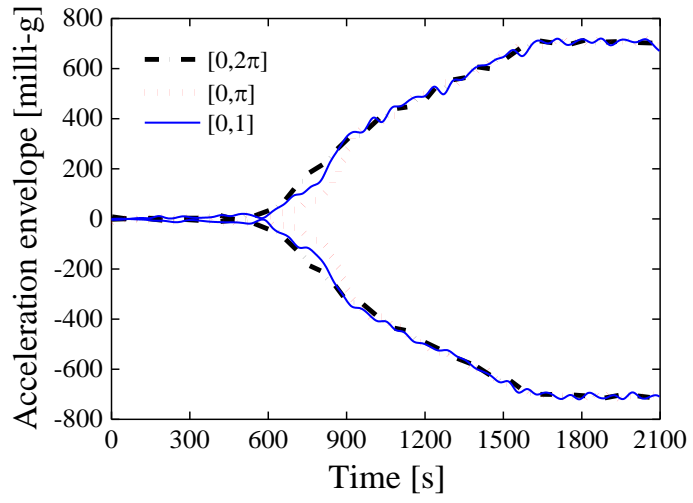

(b)

Fig. 11. Comparisons of the maximum dynamic responses at the mid-span of the bridge for three crowd initial phase distributions: (a) Lateral displacement curve, and (b) Lateral acceleration envelope curve.

\section{Table 1}

Critical numbers of pedestrians needed for different crowd initial phase distributions.

\begin{tabular}{ccccc}
\hline Uniformly distributed & \multicolumn{3}{c}{ Criterion 1 } & \multicolumn{3}{c}{ Criterion 2 } \\
\cline { 2 - 5 } interval & $\mu_{N c}$ & $\sigma_{N c}$ & $\mu_{N c}$ & $\sigma_{N c}$ \\
\hline$[0,2 \pi]$ & 156 & 25.93 & 161 & 20.19 \\
{$[0, \pi]$} & 164 & 23.96 & 166 & 18.64 \\
{$[0,1]$} & 162 & 22.18 & 170 & 16.71 \\
\hline
\end{tabular}

\subsection{Effect of crowd gait frequency distribution}

The crowd size is gradually increased from 0 to 486 pedestrians in steps of 18 every 1 minute and the crowd step length $l$ is considered to be $0.5 \mathrm{~m}$. The gait frequencies of the pedestrians $\omega_{j}$ are considered to be randomly distributed with a mean value $\mu$ and standard deviation $\sigma$. Here three different standard deviations $\sigma=0.05 \mathrm{~Hz}(0.31 \mathrm{rad} / \mathrm{s}), 0.10 \mathrm{~Hz}(0.63 \mathrm{rad} / \mathrm{s})$ and $0.15 \mathrm{~Hz}(0.94 \mathrm{rad} / \mathrm{s})$ are taken into consideration, with the same mean value $\mu=1.03 \mathrm{~Hz}(6.47 \mathrm{rad} / \mathrm{s})$. 


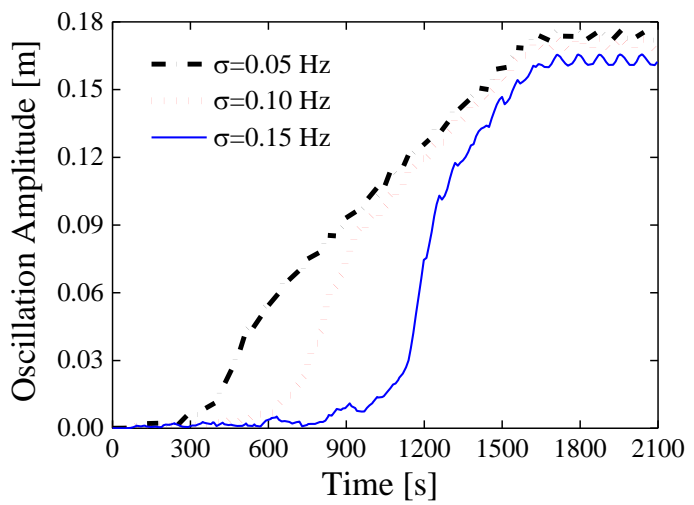

(a)

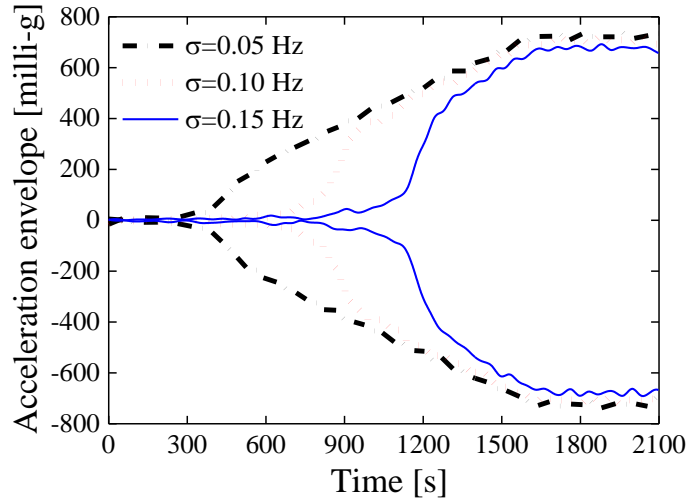

(b)

Fig. 12. Comparisons of the maximum dynamic responses at the mid-span of the bridge for three standard deviations of gait frequency: (a) Lateral displacement curve, and (b) Lateral acceleration envelope curve.

\section{Table 2}

Critical numbers of pedestrians needed for different standard deviations of gait frequency.

\begin{tabular}{ccccc}
\hline Standard deviation & \multicolumn{2}{c}{ Criterion 1 } & \multicolumn{2}{c}{ Criterion 2 } \\
\cline { 2 - 5 }$(\mathrm{Hz})$ & $\mu_{N c}$ & $\sigma_{N c}$ & $\mu_{N c}$ & $\sigma_{N c}$ \\
\hline 0.05 & 95 & 15.27 & 101 & 15.22 \\
0.10 & 156 & 25.93 & 161 & 20.19 \\
0.15 & 238 & 30.95 & 250 & 35.56 \\
\hline
\end{tabular}

Fig. 12 shows the displacement amplitude and acceleration envelope versus time curves at the mid-span of the bridge for three gait frequency distributions of the pedestrians. In fact, the standard deviation $\sigma$ controls how close the pedestrians' gait frequencies is to the crowd mean value $\mu=1.03 \mathrm{~Hz}$. Numerical results in Fig. 12 show that an increase in the standard deviation of pedestrians' gait frequencies, leads to a decrease of the maximum value of displacement or acceleration. It is clearly observed that the larger the standard deviation, the later the random gait frequencies of pedestrians become locked into the bridge frequency.

Table 2 gives the mean values $\mu_{N_{c}}$ and standard deviations $\sigma_{N_{c}}$ of the critical numbers of pedestrians for three gait frequency distributions of pedestrians under the 
two different criteria. It is seen in the second and fourth columns of Table 2 that the average critical size $\mu_{N c}$ increases significantly with an increase of the standard deviation of crowd gait frequencies $\sigma$. And it is seen in the third and fifth columns that $\sigma_{N c}$ increases with an increase of $\sigma$. In fact, the mean value $\mu_{N c}$ of Criterion 1 controls how fast pedestrians synchronize. And the standard deviation $\sigma_{N c}$ indicates the time scale at which pedestrians synchronize. The $\mu_{N c}$ of Criterion 2 is always a little larger than the corresponding value of Criterion 1.

It can be concluded from Fig. 12 and Table 2 that the homogeneity level of the normal walking frequencies of the pedestrians has a remarkable effect on the critical threshold of instability.

\subsection{Effect of pedestrian sensitivity to bridge movement}

To show the effect of pedestrian sensitivity, values of the parameter $B$ which quantifies pedestrian sensitivity to the amplitude of bridge vibration are considered in the range of $\left[0,110 \mathrm{~m}^{-1} \mathrm{~s}^{-1}\right]$. The crowd size is gradually increased from 0 to 486 in steps of 18 every 1 minute and the crowd step length $l$ is considered to be $0.5 \mathrm{~m}$ and each simulation is repeated 100 times. The results of $B$ in the range of (0-10) are not plotted in Fig. 13 because synchrony with the bridge lateral motion never occurs.

Fig. 13(a) shows the average critical numbers of pedestrians corresponding to the values of pedestrian sensitivity parameter $B$ in the interval of $\left[10 \mathrm{~m}^{-1} \mathrm{~s}^{-1}, 110 \mathrm{~m}^{-1} \mathrm{~s}^{-1}\right]$ based on both critical threshold criteria. The fitted exponential decay curves between the mean values $\mu_{N c}$ and parameter $B$ are also shown in Fig. 13(a). The corresponding fit functions for two criteria are given as follows: 


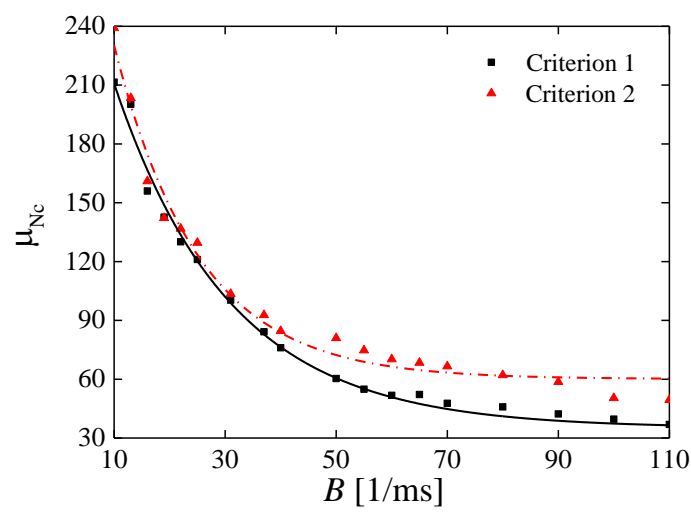

(a)

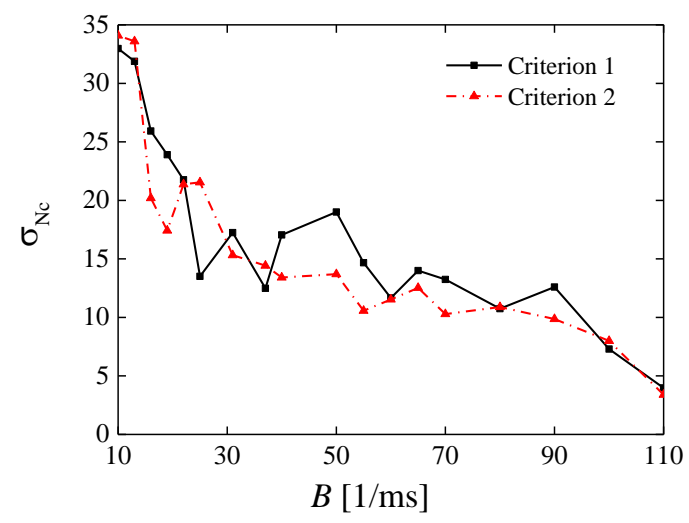

(b)

Fig. 13. The crowd critical numbers versus pedestrian sensitivity parameter curve based on two criteria: (a) Average critical number, and (b) Standard deviation.

It is observed in Fig. 13(a) that $\mu_{N c}$ for either of the two criteria generally decreases with an increase of the parameter $B$. And the mean value $\mu_{N c}$ for both criteria become steady when the parameter $B$ increases to $70 \mathrm{~m}^{-1} \mathrm{~s}^{-1}$. It is observed that when the parameter $B$ increases from $10 \mathrm{~m}^{-1} \mathrm{~s}^{-1}$ to $30 \mathrm{~m}^{-1} \mathrm{~s}^{-1}$, the average critical number $\mu_{N c}$ decreases significantly. The average critical number $\mu_{N_{c}}$ of Criterion 2 is always a little larger than that of Criterion 2.

Fig. 13(b) displays the relationships between the standard deviations $\sigma_{N c}$ associated to the average critical numbers of pedestrians $\mu_{N c}$ and parameter $B$ within $\left[10 \mathrm{~m}^{-1} \mathrm{~s}^{-1}\right.$, $\left.110 \mathrm{~m}^{-1} \mathrm{~s}^{-1}\right]$. It is seen in Fig. 13(b) that the standard deviation $\sigma_{N c}$ generally decreases with an increase of the parameter $B$. When the parameter $B$ increases from $10 \mathrm{~m}^{-1} \mathrm{~s}^{-1}$ to $30 \mathrm{~m}^{-1} \mathrm{~s}^{-1}, \sigma_{N c}$ decreases most significantly.

In fact, for low $B$ values, the pedestrians are less susceptible to bridge movement, which means crowd initial behavior is more random. Consequently, this randomness 
has a remarkable effect on the critical threshold of instability. When the sensitivity increases, the pedestrians are driven to change their footsteps to be locked into the bridge motion quicker. Therefore, the crowd critical size for high $B$ value is lower than that for low $B$ value.

\subsection{Effect of bridge frequency}

The crowd size is gradually increased from 0 to 486 pedestrians in steps of 18 every 1 minute and the crowd step length $l$ is considered to be $0.5 \mathrm{~m}$. A parameter $\beta$ is introduced to represent the ratio of the mean value of the gait frequencies $\mu$ to the bridge frequency $\omega_{0} . \beta=\mu / \omega_{0}=0.90,0.95,1.00$ and 1.05 are evaluated.

Fig. 14 compares the maximum lateral displacement-time and acceleration-time histories at the mid-span of the bridge for the four values of $\beta$. It can be seen from these responses that an increase of the ratio $\beta$ leads to the increase of the maximum final value of displacement or acceleration. It is also seen that the emergence of the synchronization will generally speed up an increase of the ratio $\beta$.

Table 3 gives the mean values $\mu_{N c}$ and standard deviations $\sigma_{N c}$ of the critical numbers of pedestrians for the four ratios (denoted as $\beta=\mu / \omega_{0}$ ) under two different criteria. It can be seen in the second and fourth columns of Table 4 that the value $\mu_{N c}$ decreases notably with the increase of the ratio $\beta$. The crowd critical size $\mu_{N c}$ for Criterion 2 is always slightly larger than the corresponding value Criterion 1 . 


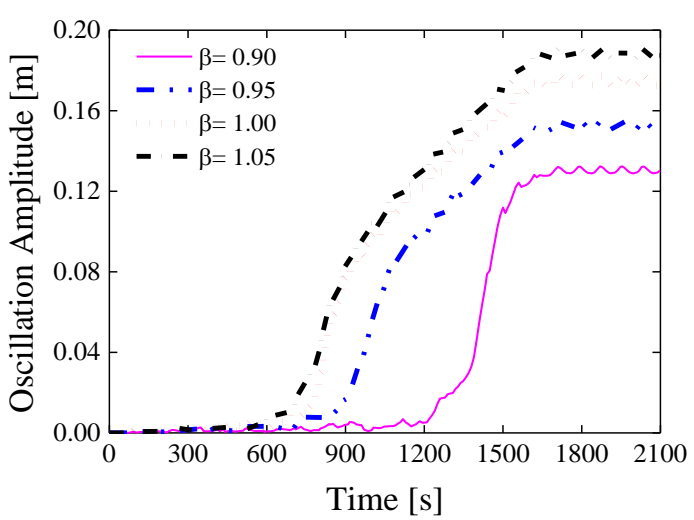

(a)

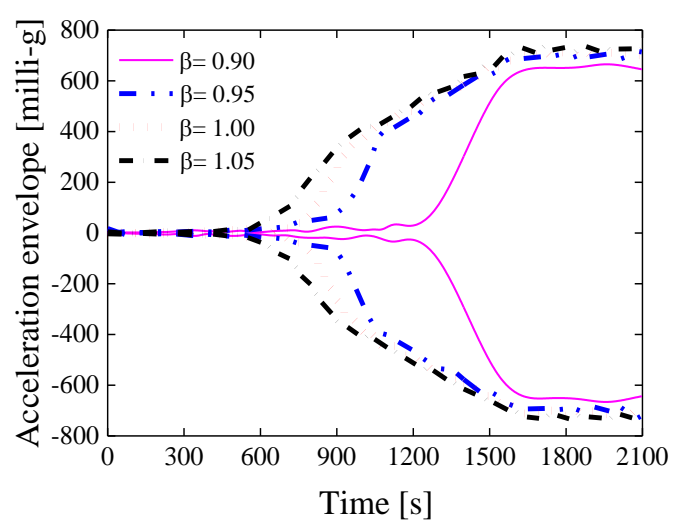

(b)

Fig. 14. Comparisons of the maximum dynamic responses at the mid-span of the bridge for four average pedestrian frequency to bridge frequency ratios: (a) Lateral displacement curve, and (b) Lateral acceleration envelope curve.

\section{Table 3}

Critical numbers of pedestrians for different average pedestrian frequency to bridge frequency ratios.

\begin{tabular}{ccccc}
\hline \multirow{2}{*}{$\beta=\mu / \omega_{0}$} & \multicolumn{2}{c}{ Criterion 1 } & \multicolumn{2}{c}{ Criterion 2 } \\
\cline { 2 - 5 } & $\mu_{N c}$ & $\sigma_{N c}$ & $\mu_{N c}$ & $\sigma_{N c}$ \\
\hline 0.90 & 268 & 41.75 & 283 & 36.94 \\
0.95 & 195 & 33.36 & 199 & 28.02 \\
1.00 & 156 & 25.93 & 161 & 20.19 \\
1.05 & 144 & 32.31 & 158 & 26.94 \\
\hline
\end{tabular}

It is concluded from the above results that the fundamental lateral frequency of the bridge should be designed to be larger than the dominant walking frequency of pedestrians, for safety reasons.

\subsection{Effect of bridge damping}

The number of pedestrians on the bridge increases from 0 to 486 pedestrians in steps of 18 every 1 minute and the crowd step length $l$ is considered to be $0.5 \mathrm{~m}$, as an example. Eight values of bridge damping ratios $\zeta$ varying from $0.75 \%$ to $2.50 \%$ are considered. It should also be noted that energy exchange between the crowd and the moving surface is not considered here. 


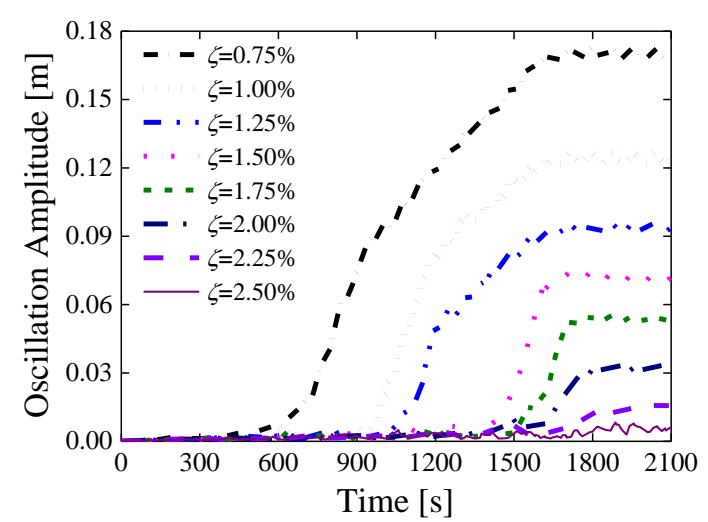

(a)

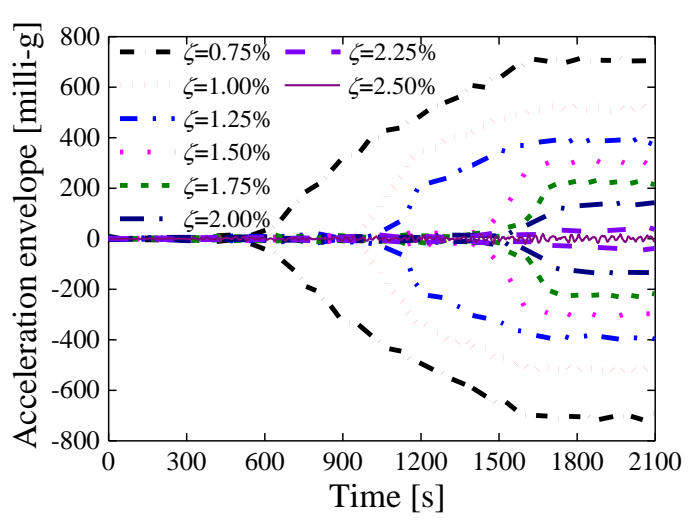

(b)

Fig. 15. Comparisons of the maximum dynamic responses at the mid-span of the bridge for eight bridge damping ratios: (a) Lateral displacement curve, and (b) Lateral acceleration envelope curve.

\section{Table 4}

Critical numbers of pedestrians needed for six bridge damping ratios based on two criteria.

\begin{tabular}{ccccc}
\hline \multirow{2}{*}{$\zeta(\%)$} & \multicolumn{2}{c}{ Criterion 1 } & \multicolumn{2}{c}{ Criterion 2 } \\
\cline { 2 - 5 } & $\mu_{N c}$ & $\sigma_{N c}$ & $\mu_{N c}$ & $\sigma_{N c}$ \\
\hline 0.75 & 156 & 25.93 & 161 & 20.19 \\
1.00 & 213 & 28.95 & 218 & 29.73 \\
1.25 & 249 & 30.08 & 263 & 35.17 \\
1.50 & 300 & 33.69 & 316 & 33.28 \\
1.75 & 338 & 35.35 & 356 & 37.34 \\
2.00 & 390 & 29.95 & 412 & 29.94 \\
\hline
\end{tabular}

Fig. 15 compares the maximum lateral displacements and accelerations at the mid-span of the bridge for eight bridge damping ratios $\zeta$ varying from $0.75 \%$ to $2.50 \% . \zeta=0.75 \%$ represents the damping ratio of the fundamental mode of the LMF before installing any damping devices. Numerical results in Fig. 15 show that an increase of the bridge damping ratio results in a significant decrease of the magnitude of structural response. It is also observed that the emergence of the accumulated synchronization will generally slow down with an increase of the bridge damping ratio. When the value of $\zeta$ reaches $2.25 \%$, synchrony with the bridge lateral motion will not occur. 
Table 4 lists the mean values $\mu_{N c}$ and standard deviations $\sigma_{N c}$ of the critical numbers of pedestrians needed for six bridge damping ratios based on two different criteria. The critical thresholds when the bridge damping ratios are $2.25 \%$ and $2.50 \%$ are not given. This is because pedestrians are never able to synchronize in these two situations and the bridge response remains confined to small oscillations. It is seen in the second and fourth columns of Table 4 that the average critical size $\mu_{N c}$ increases significantly with an increase of the bridge damping ratio $\zeta$. The value of $\mu_{N c}$ for Criterion 2 is always a little larger than that needed for Criterion 1.

In modern footbridge design, adding additional dampers can increase not only the bridge damping ratio, but also the natural frequency of the bridge. Therefore, it is an efficient measurement to reduce the vibration and control the dynamic stability of the structure.

\section{Conclusions}

Based on the coupling oscillator theory, a new model of bi-directional walking pedestrians-bridge interaction is proposed. The pedestrians during walking are modelled as lateral footstep forces acting on the bridge, which are not affected by the bridge motion. The real case of the London Millennium Footbridge is studied to examine the applicability and the correctness of the present model. Parametric studies of the bridge lateral vibrations are conducted. Two criteria are adopted to determine the critical number of pedestrians. From the above study, the following conclusions can be drawn:

1. In the present model, pedestrians enter the bridge from two ends, which is more 
realistic than one-way walking. The contribution of the lateral force varies with pedestrians' moving positions. The present model is shown to successfully predict the lateral responses and the critical threshold of the north span of the London Millennium Footbridge, compared with observations reported from its opening day.

2. From the parameter scopes study, the step length and the initial level of phase synchrony among pedestrians have little effect on the dynamic response of the bridge and the critical numbers of pedestrians for the two criteria. The pedestrian sensitivity to bridge motion and the combination of step size and duration of the load event both play an important role in the critical numbers of pedestrians for the two criteria.

3. The homogeneity level of normal walking frequencies of pedestrians has a remarkable impact on the response characteristics of the bridge and the critical threshold of instability. It may also affect the size of the time scale in which pedestrians will synchronize with the bridge sway.

4. Different average pedestrian frequency to bridge frequency ratios and bridge damping ratios will affect both the dynamic response of the event and the critical numbers of pedestrians triggering excessive vibrations. Therefore, adding additional dampers and increasing the bridge frequency to a value well above the dominant walking frequency of pedestrians are efficient measures for reducing vibration and controlling the dynamic stability of the structure.

5. The mean value of the critical crowd number obtained will be different depending 
on the different criteria for instability. In the parameter scopes study, the critical threshold needed to exceed the acceleration threshold $0.2 \mathrm{~m} / \mathrm{s}^{2}$ is always a bit larger than that for the onset of excessive lateral vibration.

\section{Acknowledgements}

The authors are grateful to Dr. Brian Ellis for his comments and corrections. The financial support from the National Natural Science Foundation of China (11372127; 51778289) and the Key Program of Natural Science Research of Jiangsu Provincial University (12KJA580002) are greatly acknowledged. This work is also supported in part by the scholarship from China Scholarship Council (CSC) under the Grant No. 201708320368.

\section{References}

[1] D. Lynch, Bridge away feared cause of Cambodian festival tragedy, New Civil Engineer, 2010.

[2] Y. Fujino, B. M. Pacheco, S.I. Nakamura, P. Warnitchai, Synchronization of human walking observed during lateral vibration of a congested pedestrian bridge, Earthq. Eng. Struct. D. 22 (9) (1993) 741-758.

[3] E.T. Ingólfsson, C.T. Georgakis, J. Jönsson, Pedestrian-induced lateral vibrations of footbridges: a literature review, Eng. Struct. 45 (2012) 21-52.

[4] J.M.W. Brownjohn, P. Fok, M. Roche, P. Omenzetter, Long span steel pedestrian bridge at Singapore Changi Airport - part 2: Crowd loading tests and vibration mitigation measures, Struct. Engr. 82 (16) (2004) 28-34.

[5] J.H.G. Macdonald, Pedestrian-induced vibrations of the Clifton Suspension Bridge, UK, Proceedings of the Institution of Civil Engineers — Bridge Engineering, 161 (2008) pp. 6977.

[6] P. Dallard, A.J. Fitzpatrick, A. Flint, S.L. Bourva, A. Low, R.M.S. Ridsdill, M. Willford, The London Millennium Footbridge, Struct. Engr. 79 (22) (2001) 17-33.

[7] F. Venuti, L. Bruno, Crowd-structure interaction in lively footbridges under synchronous lateral excitation: a literature review, Phys. Life. Rev. 6 (2009) 176-206.

[8] H.X. Han, D. Zhou, T. Ji, Mechanical parameters of standing body and applications in human-structure interaction, Int. J. Appl. Mech. 9 (2) (2017) 1750021-1-1750021-30.

[9] Y.A. Gao, Q.S. Yang, Y. Dong, A three-dimensional pedestrian-structure interaction model for general applications, Int. J. Struct. Stab. Dy. 18 (9) (2018) 1850107-1-1850107-27.

[10] S. Živanovic, A. Pavic, P. Reynolds, Vibration serviceability of footbridges under 
human-induced excitation: a literature review, J. Sound. Vib. 279 (2005) 1-74.

[11] V. Racic, A. Pavic, J.M.W. Brownjohn, Experimental identification and analytical modeling of human walking forces: Literature review, J. Sound. Vib. 326 (2009) 1-49.

[12] Y. Fujino, M. S. Dionysius, A conceptual review of pedestrian-induced lateral vibration and crowd synchronization problem on footbridges, J. Bridge. Eng. 21 (8) (2016) C4015001-1C4015001-12.

[13] C. Barker, Some observations on the nature of the mechanism that drives the self-excited lateral response of footbridges, Footbridge 2002- First International Conference, Paris, Friance, (2002) pp.1-9.

[14] J.H.G. Macdonald, Lateral excitation of bridges by balancing pedestrian, Proceedings of the Royal Society A-Mathematical, Physical and Engineering Sciences 465 (2008) 1055-1073.

[15] M. Bocian, J.H.G. Macdonald, J.F. Burn, Biomechanically inspired modeling of pedestrian-induced forces on laterally oscillating structures, J. Sound. Vib. 331 (2012) 39143929.

[16] G.H. Goldsztein, Lateral oscillations of the center of mass of bipeds as they walk. Inverted pendulum model with two degrees of freedom, AIP. Adv. 5 (10) (2015) 107208-1-107208-10.

[17] I. Belykh, R. Jeter, V. Belykh, Foot force models of crowd dynamics on a wobbly bridge, Sci. Adv. 3 (11) (2017) e1701512.

[18] E.T. Ingólfsson, C.T. Georgakis, F. Ricciardelli, J. Jönsson, Experimental identification of pedestrian-induced lateral forces on footbridges, J. Sound. Vib. 330 (2011) 1265-1284.

[19] S.P. Carroll, J.S. Owen, M.F.M. Hussein, Reproduction of lateral ground reaction forces from visual marker data and analysis of balance response while walking on a laterally oscillating deck, Eng. Struct. 49 (2013) 1034-1047.

[20] M. Bocian, J.H.G. Macdonald, J.F. Burn, D. Redmill, Experimental identification of the behavior of and lateral forces from freely-walking pedestrinas on laterally oscillating structures in a virtual reality environment, Eng. Struct. 105 (2015) 62-76.

[21] M. Bocian, J.F. Burn, J.H.G. Macdonald, J.M.W. Brownjohn, From phase drift to synchronization-pedestrian stepping behavior on laterally oscillating structures and consequences for dynamic stability, J. Sound. Vib. 392 (2017) 382-399.

[22] D. Claff, M.S. Williams, A. Blakeborough, The kinematics and kinetics of pedestrians on a laterally swaying footbridge, J. Sound. Vib. 407 (2017) 286-308.

[23] K.V. Nimmen, G. Lombaert, I. Jonkers, G.D. Roeck, P.V.D. Broeck, Characterisation of walking loads by 3D inertial motion tracking, J. Sound. Vib. 333 (2014) 5212-5226.

[24] J.M.W. Brownjohn, J. Chen, M. Bocian, V. Racic, E. Shahabpoor, Using inertial measurement units to identify medio-lateral ground reaction forces due to walking and swaying, J. Sound. Vib. 426 (2018) 90-110.

[25] S. Nakamura, Model for lateral excitation of footbridges by synchronous walking, J. Struct. Eng. 130 (1) (2004) 32-37.

[26] D.E. Newland, Pedestrian excitation of bridges, Proceedings of the Institution of Mechanical Engineers Part C - Journal of Mechanical Engineering Science 218 (2004) 477-492.

[27] T.M. Roberts, Lateral pedestrian excitation of footbridges, J. Bridge. Eng. 10 (2005) 107112.

[28] S.H. Strogatz, D.M. Abrams, A. McRobie, B. Eckhardt, E. Ott, Crowd synchrony on the Millennium Bridge, Nature 438 (2005) 43-44. 
[29] D.M. Abrams, Two coupled oscillator models: the Millennium Bridge and the chimera state [Ph.D. Thesis]. Ithaca (New York): Faculty of the Graduate School, Cornell University; 2006.

[30] Video footages for Arup tests on London's Millennium Bridge; 2001. www.arup.com/millenniumbridge/indepth/video.html. Accessed 21 May 2017 from Professor D.M. Abrams.

[31] F. Gazzola, V. Racic, A model of synchronization in crowd dynamics, App. Math. Model. 59 (2018) 305-318.

[32] M.A. Toso, H.M. Gomes, A coupled biodynamic model for crowd-footbridge interaction, Eng. Struct. 177 (2018) 47-60.

[33] V. Racic, J.M.W. Brownjohn, Mathematical modeling of random narrow band lateral excitation of footbridges due to pedestrians walking, Comput. Struct. 90-91 (2012) 116-130.

[34] A.K. Chopra, Dynamic of structures: theory and applications to earthquake engineering, Prentice-Hall, 2001.

[35] A. McRobie, G. Morgenthal, J. Lasenby, M. Ringer, Section model tests on human-structure lock-in, Bridge. Eng. 156 (2) (2003) 71-79.

[36] A. Pachhi, T. Ji, Frequency and velocity of people walking, Struct. Engr. 84 (3) (2005) 3640.

[37] B.R. Ellis, Serviceability evaluation of floor vibration induced by walking loads, Struct. Engr. 79 (21) (2001) 30-36.

[38] L. Marcheggiani, S. Lenci, On a model for the pedestrians-induced lateral vibrations of footbridges, Meccanica 45 (4) (2010) 531-551.

[39] D. Sudjic, Blade of light: the story of London's Millennium Bridge, Penguin press, 2001.

[40] Sétra, Footbridges - Assessment of vibrational behaviour of footbridges under pedestrian loading, SETRA/AFGC, French Association of Civil Engineering, 2006.

[41] E.T. Ingólfsson, C.T. Georgakis, A stochastic load model for pedestrian-induced lateral forces on footbridges, Eng. Struct. 33 (12) (2011) 3454-3470. 